\title{
Molecular Orbital Study of the Hydrogen Bond in Ice
}

Mariana Weissmann, and Norah V. Cohan

Citation: The Journal of Chemical Physics 43, 119 (1965); doi: 10.1063/1.1696438

View online: https://doi.org/10.1063/1.1696438

View Table of Contents: http://aip.scitation.org/toc/jcp/43/1

Published by the American Institute of Physics

\section{Articles you may be interested in}

Molecular Orbital Studies of Hydrogen Bonds. III. $\mathrm{C}=\mathrm{O} \cdots \mathrm{H}-\mathrm{O}$ Hydrogen Bond in $\mathrm{H}_{2} \mathrm{CO} \cdots \mathrm{H}_{2} \mathrm{O}$ and $\mathrm{H}_{2} \mathrm{CO} \cdots 2 \mathrm{H}_{2} \mathrm{O}$

The Journal of Chemical Physics 55, 1236 (1971); 10.1063/1.1676210

Perspective: Found in translation: Quantum chemical tools for grasping non-covalent interactions The Journal of Chemical Physics 146, 120901 (2017); 10.1063/1.4978951

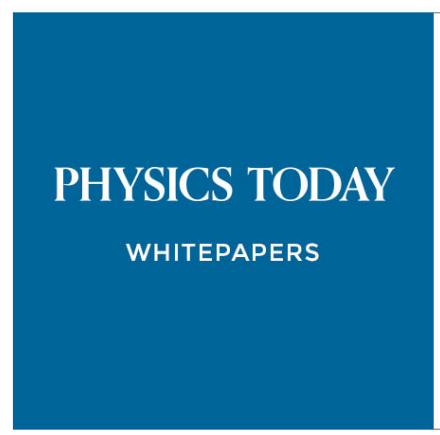

ADVANCED LIGHT CURE ADHESIVES

Take a closer look at what these environmentally friendly adhesive systems can do
READ NOW

PRESENTED BY

(8) MASTERBOND 


\title{
Molecular Orbital Study of the Hydrogen Bond in Ice
}

\author{
Martana Weissmann \\ Facultad de Ciencias Exactas y Naturales, Perú 272, Buenos Aires, Argentina \\ AND \\ Norah V. COHAN* \\ Facultad de Ciencias Exactas y Naturales, Perü 272, Buenos Aires, Argentina, and \\ Consejo Nacional de Investigaciones Cientificas y Técnicas, Buenos Aires, Argentina
}

(Received 17 November 1964)

\begin{abstract}
An SCF-MO-LCAO calculation for the four electrons involved in the straight hydrogen bond in ice is performed using a limited set of Slater-type orbitals. The energy of the four electrons plus the interaction between the "core potentials" is calculated as a function of the position of the proton along the line joining the oxygens. The energy of the hydrogen bond is obtained $-8.2 \mathrm{kcal} / \mathrm{mole}$-in good agreement with experiment. Electrostatic and delocalization contributions to the energy of the hydrogen bond are in excellent agreement with previous theoretical estimates. The potential energy for the motion of the proton results in a very asymmetric curve, with only one minimum. The dipole moment increases from $1.68 \mathrm{D}$ for a free water molecule to $2.40 \mathrm{D}$ thus confirming previous estimates.
\end{abstract}

\section{INTRODUCTION}

$\mathbf{T}$ HE hydrogen bond has received considerable attention in the last few years, ${ }^{1}$ and good semiempirical potential-energy functions have been proposed $^{2}$ to account for several of the relevant properties of the hydrogen-bonded systems. But from the theoretical point of view very few nonempirical calculations have been made. ${ }^{3}$ In particular, the importance of the different contributions to the energy of the hydrogen bond has been pointed out lately, ${ }^{4}$ and all the contributions mentioned-electrostatic, delocalization, repulsion, and dispersion - seem to be of the same order of magnitude. It then seems reasonable that some sort of $a b$ initio quantum-mechanical calculation, which would include all these effects, or at least the most important part of them, is worthwhile performing. Finally, it is also of interest to obtain a theoretical estimate of the asymmetric potential for the motion of the proton in a single hydrogen bond and to compare it with the semiempirical proposed ones.

In the present work, an SCF-MO-LCAO calculation for the four electrons involved in the straight hydrogen bond in ice, is performed. The crystal structure of ice is well known, ${ }^{1}$ each oxygen being surrounded by four others in approximately tetrahedral directions. In the line between two oxygens there is only one hydrogen. A limited set of Slater-type basic orbitals is used, and the calculations are done for different values of the distances involved. The energy of the hydrogen bond

\footnotetext{
* Now at Department of Chemistry, University of Pennsylvania, Philadelphia, Pennsylvania.

1 G. C. Pimentel and A. L. McClellan, The Hydrogen Bond (W. H. Freeman and Company, San Francisco, 1960).

2 E. E. Lippincott and R. Schroeder, J. Chem. Phys. 23, 1099 (1955); J. Phys. Chem. 61, 921 (1957); C. Reid, J. Chem. Phys. 30, 182 (1959).

${ }^{3}$ C. A. Coulson and U. Danielsson, Arkiv. Fysik 8, 239, 245 (1954); H. Tsubomura, J. Chem. Phys. 23, 2130 (1955); 24, 927 (1956); P. C. McKinney and G. M. Barrow, ibid. 31, 294 (1959); L. Paoloni, ibid. 30, 1045 (1959)

${ }^{4}$ C. A. Coulson, Research 10, 149 (1957).
}

is obtained, as well as the potential-energy curve for the motion of the proton along the $\mathrm{O}-\mathrm{O}$ line. Some other properties are also obtained as byproducts of the calculations; in particular the increase in dipole moment due to hydrogen bonding is computed.

\section{BASIC SET OF ORBITALS}

For the oxygen atoms only $2 s$ and $2 p$ orbitals have been considered; $1 s$ electrons are not included in the

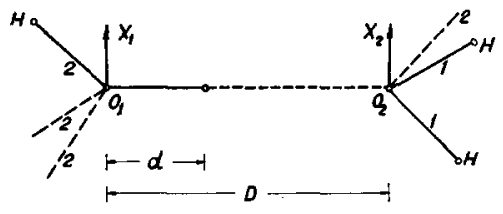

Fig. 1. Two complete water molecules forming a hydrogen bond. The numbers on the bonds indicate the occupation number of the corresponding tetrahedral hybrids.

calculations so that the oxygen cores are given positive charges of $6 e$.

Straight bonds have been considered throughout although it is known that in gaseous water the $\mathrm{OH}$ bonds are slightly "bent." ${ }^{\prime}$ Therefore no contributions from $2 p_{\pi}$ orbitals of the oxygens perpendicular to the $\mathrm{O}_{1}-\mathrm{H}-\mathrm{O}_{2}$ line need be considered (see Fig. 1). Only the $1 s$ orbital of hydrogen was included. Therefore there are five basic orbitals: $2 s$ and $2 p_{\sigma}$ orbitals on the two oxygens and the $1 s$ orbital on the hydrogen. Slater-type atomic orbitals with orbital exponent 2.275 for the $n=2$ orbitals and 1 for the hydrogen $1 s$ orbital were used.

The results obtained by a SCF procedure with the above set of five orbitals are obviously absurd, as the resulting hydrogen bond would be inequivalent to the other hydrogen bonds. To avoid this situation, we have

${ }^{5}$ F. O. Ellison and H. Shull, J. Chem. Phys. 23, 2348 (1955); R. McWeeny and K. A. Ohno, Proc. Roy. Soc. (London) A255, 367 (1960). 
replaced the $2 s$ and $2 p_{\sigma}$ orbitals on each oxygen by a tetrahedral hybrid pointing towards the other oxygen.

The notation used for the orbitals is the following: $s_{i}, x_{i}, z_{i}$, and $h_{i}$ are the $2 s, 2 p_{x}, 2 p_{z}$, and hybrid orbitals, and the suffix $i$ being 1 or 2 according to whether the orbitals are centered in Nucleus $\mathrm{O}_{1}$ or $\mathrm{O}_{2}$. The $1 s$ orbital of hydrogen is called $h$.

\section{3. "CORE" POTENTIAL}

The Hartree-Fock Hamiltonian $F$ of Electron $i$ is, as usual,

$$
F(i)=H(i)+\sum_{k=1}^{2}\left[2 J_{k}(i)-K_{k}(i)\right],
$$

where $J_{j}$ and $K_{j}$ are the Coulomb and exchange operators and the summation is over the two lowest MO.

$H$, the so-called "core" Hamiltonian, is the sum of the kinetic-energy operator and the "core" potential field. This core potential field is made up by the three nuclei with charges +6 on the oxygens $\mathrm{O}_{1}$ and $\mathrm{O}_{2}$ and +1 on the hydrogen and the rest of the electrons of the two oxygens, not involved in the bond. These electrons are assumed to be in the other three tetrahedral hybrids of each oxygen, these hybrids being singly or doubly occupied according to whether they correspond to $\mathrm{O}-\mathrm{H}$ bonds or lone pairs, respectively. Thus, in the "core" potential, the effect of the hydrogens and atoms further apart are neglected. The "core" potential thus used is not self-consistent, as later (see Table II) it is shown that there is some delocalization of charge as well as changes in the polarity of the $\mathrm{O}-\mathrm{H}$ bonds due to hydrogen bonding. Therefore, a self-consistent "core" potential should include all the atoms involved in the six neighbor hydrogen bonds, thus making the calculations extremely cumbersome.

Therefore, in atomic units:

$$
H=H(i)=-\frac{1}{2} \nabla_{i}^{2}-\sum_{j=1}^{2} \frac{6}{r_{i}\left(\mathrm{O}_{j}\right)}-\frac{1}{r_{i H}}+\sum_{j=1}^{2} \rho_{j}(i),
$$

where

$$
\begin{aligned}
\rho_{j}(i)=\frac{1}{4} Q_{j}\left[S_{j}(i)\right. & S_{j}(i)+\frac{1}{3} z_{j}(i) z_{j}(i) \\
& \left.+\frac{8}{3} x_{j}(i) x_{j}(i)-(2 / \sqrt{3}) S_{j}(i) z_{j}(i)\right],
\end{aligned}
$$

and $Q_{j}=5$ for $j=1$ and $Q_{j}=4$ for $j=2$. Therefore, $Q_{j}$ represents the total electronic charge, on Atom $\mathrm{O}_{j}$, not involved in the hydrogen bond. In Fig. 1 a schematic representation of the two complete water molecules and of the occupation number of the hybrids is given.

It should be remembered that in ice there are two possible orientations for two water molecules: trans and cis, in the ratio 3 to 1 . Only the trans orientation was considered, in accordance with some previous results. ${ }^{6}$ The different orientations of the hydrogen atoms within the trans configuration of the molecules were considered and properly averaged.

\footnotetext{
${ }^{6}$ N. V. Cohan, M. Cotti, J. V. Iribarne, and M. Weissmann, Trans. Faraday Soc. 58, 490 (1962)
}

\section{CALCULATIONS}

The total energy of the system $E=E_{\mathrm{e} 1}+E_{\text {int }}$ is made up of the electronic energy of the system of four electrons, $E_{\mathrm{el}}$, and of the interaction between the "cores," $E_{\text {int }}$. A closed-shell configuration was assumed for the four electrons; thus:

$$
E_{\mathrm{el}}=2 \sum_{i=1}^{2} H_{i}+\sum_{i, j=1}^{2}\left(2 J_{i j}-K_{i j}\right)
$$

where, as usual, $H_{i}=\left\langle\phi_{i}|H| \phi_{i}\right\rangle, J_{i j}=\left\langle\phi_{i}\left|J_{j}\right| \phi_{i}\right\rangle$, and $K_{i j}=\left\langle\phi_{i}\left|K_{j}\right| \phi_{i}\right\rangle$. The $\phi_{i}$ 's are the SCF-MO$\mathrm{LCAO}$, and are calculated by Roothaan's ${ }^{7}$ SCF procedure.

$$
\begin{aligned}
E_{\mathrm{int}}=\frac{6}{d} & +\frac{6}{D-d}+\frac{36}{D}-\sum_{j=1}^{2} \int \frac{\rho_{j}(i)}{r_{i H}} d \tau_{i} \\
& -6 \sum_{j=1}^{2} \int \frac{\rho_{j}(i)}{r_{i}\left(\mathrm{O}_{j}\right)} d \tau_{i}+\iint \frac{\rho_{1}(i) \rho_{2}(i)}{r_{12}} d \tau_{1} d \tau_{2}
\end{aligned}
$$

and is calculated directly. The $\rho_{j}(i)$ are defined in (3), and the distances are shown in Fig. 1.

All one-electron integrals and two-electron Coulomb integrals were calculated with Roothaan's ${ }^{8}$ formulas. Exchange and hybrid integrals were kindly computed for us by the Laboratory of Molecular Structure and Spectra of the University of Chicago. Three-center integrals were approximated by Mulliken's procedure ${ }^{9}$ The larger three-center integrals, of the hybrid type, were also programmed in a few cases using Zauli and Magnusson's ${ }^{10}$ method. Although the difference in the values of the integrals obtained by the two procedures was significant, its effect on the final results is not important.

The integrals, the SCF calculation, and the calculation of $E_{\text {int }}$ were all programmed for the Ferranti Mercury Computer of the Instituto de Cálculo of the University of Buenos Aires.

\section{RESULTS AND DISCUSSION}

The total energy $E$ calculated as a function of $d$ represents the potential energy for the motion of the proton along the $\mathrm{O}_{1}-\mathrm{O}_{2}$ line. The results for $E$ as a function of the distances $D$ and $d$ are given in Table I and in Fig. 2.

As expected, the curves of Fig. 2 are strongly asymmetric, but no second minimum is observed. Although very much has been written about the existence of this second minimum, it seems to some authors ${ }^{4,11}$ and it is our own view also, that this is not essential to explain the properties of ice. In particular, Somorjai and

${ }^{7}$ C. C. J. Roothaan, Rev. Mod. Phys. 23, 69 (1951).

${ }^{8}$ C. C. J. Roothaan, J. Chem. Phys. 19, 1445 (1951).

${ }^{9}$ R. S. Mulliken, J. Chim. Phys. 46, 500, 521 (1949).

10 E. A. Magnusson and C. Zauli, Proc. Phys. Soc. (London) 78, 53 (1961).

it R. L. Somorjai and D. F. Hornig, J. Chem Phys. 36, 1980 (1962). 
TABLE I. Total energy (in a.u.) for different values of $d$ and $D$ (in atomic units). The last line in each case corresponds to the minimum value of $E$, fitted numerically. ( 1 a.u. of energy $=27.2 \mathrm{eV}, 1$ a.u. of length $=0.529 \AA$.)

\begin{tabular}{|c|c|c|c|c|c|c|c|}
\hline \multicolumn{2}{|c|}{$D=4.7$} & \multicolumn{2}{|c|}{$D=5.2154$} & \multicolumn{2}{|c|}{$D=5.7$} & \multicolumn{2}{|c|}{$D=\infty$} \\
\hline$d$ & $E$ & $d$ & $E$ & $d$ & $E$ & $d$ & $E$ \\
\hline $\begin{array}{l}1.0029 \\
1.4116 \\
1.8103 \\
2.2090 \\
2.3500 \\
2.4910 \\
2.8897 \\
3.2884 \\
3.6971 \\
1.5808\end{array}$ & $\begin{array}{l}-3.568 \\
-3.999 \\
-3.993 \\
-3.906 \\
-3.875 \\
-3.846 \\
-3.771 \\
-3.642 \\
-3.126 \\
-4.028\end{array}$ & $\begin{array}{l}1.0029 \\
1.4116 \\
1.8103 \\
2.2090 \\
2.6077 \\
3.0064 \\
3.4051 \\
3.8038 \\
4.2125 \\
1.5741\end{array}$ & $\begin{array}{l}-3.559 \\
-3.988 \\
-3.977 \\
-3.880 \\
-3.783 \\
-3.712 \\
-3.659 \\
-3.547 \\
-3.039 \\
-4.016\end{array}$ & $\begin{array}{l}1.4116 \\
1.8103 \\
2.2090 \\
2.6077 \\
2.8500 \\
3.0923 \\
3.4910 \\
3.8897 \\
4.2884 \\
1.5711\end{array}$ & $\begin{array}{l}-3.982 \\
-3.969 \\
-3.865 \\
-3.757 \\
-3.702 \\
-3.657 \\
-3.607 \\
-3.572 \\
-3.475 \\
-4.009\end{array}$ & $\begin{array}{c}1.0029 \\
1.4116 \\
1.8103 \\
2.6077 \\
3.4051 \\
4.2125 \\
\infty\end{array}$ & $\begin{array}{l}-3.544 \\
-3.973 \\
-3.957 \\
-3.727 \\
-3.524 \\
-3.384 \\
-2.837\end{array}$ \\
\hline
\end{tabular}

Hornigil have shown that it is highly improbable that the broadening in the ir spectra, very often attributed to the double minimum, is actually due to it. It should be mentioned here that our curves represent the motion of one proton in a single hydrogen bond. It might be possible that in ice and other systems, as suggested for example by Reid, ${ }^{2}$ a collective motion of protons along several hydrogen bonds takes place, thus producing important changes in the shape of the potential-energy curves.

We also find that the potential-energy curves become more symmetric, and the distance $d$ increases slightly, as $D$ decreases. These results are in qualitative agreement with experiment and semiempirical models, ${ }^{12,2}$ although the calculated effects are too small. The minimum value of the energy corresponds to a value of $D$, not precisely determined, but surely about $0.5 \AA$ smaller than the experimental value in ice, $D=2.76 \AA(5.2154$ a.u.). We believe that the main reason for obtaining the optimum value of $D$ too small, and also a small

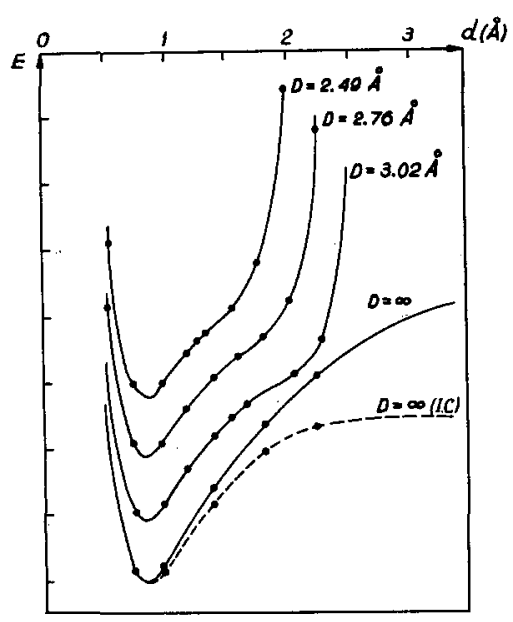

FIG. 2. Total energy as function of the distance $d$, for four values of $D$. The intervals marked on the energy scale are of 0.2 a.u. $=5.44 \mathrm{eV}$, and each curve is shifted vertically from the preceding one by exactly that amount. I.C. means configuration interaction.

${ }^{12}$ K. Nakamoto, M. Margoshes, and R. E. Rundle, J. Am. Chem. Soc. 77, 6480 (1955). variation of $d$ with $D$, is our neglecting the deformation of the "core" potential as $D$ decreases. Another important effect that was neglected is the exchange with other electrons, that is, our keeping the problem to a four-electron system.

The optimum value of $d$ for the experimental value of $D$ is $d=0.83 \AA$ (1.5808 a.u.), thus being about $0.2 \AA$ too short in comparison with experiment. ${ }^{13}$ The neglected effects mentioned above might also contribute to the error found for the value of $d$. But it should also be remembered that we have used an extremely limited set of basic orbitals in our SCF treatment, with Slater orbitals and orbital exponents, which is surely a very poor basis. For the case $D=\infty$ configuration interaction with another closed-shell configuration, which allows for dissociation into neutral fragments as $d$ tends to $\infty$, was tried and proved useless in improving the optimum value of $d$ (see Fig. 2). Therefore it was not attempted for other values of $D$ as the work involved is considerably greater.

All the following results in this paper, if not stated otherwise, are given for $D=2.76 \AA$ (5.2154 a.u.) and $d=0.96 \AA$ (1.8103 a.u.), this last value being the experimental one in free water. It was used only for convenience in the calculations, and the results below will alter only slightly if the experimental value for ice $d=1.01 \AA^{13}$ is used. We have used the experimental values of the distances rather than those obtained by minimizing the total energy $E$ because all the properties calculated for the experimental distances are in reasonable agreement with experiment or with the results of other calculations. If our too small values of $D$ and $d$ were used, all the results--and in particular the energy of the hydrogen bond-depart considerably from the experimental values.

The curve labeled $D=\infty$ in Fig. 2 corresponds to the energy of a two-electron system representing one $\mathrm{O}-\mathrm{H}$ bond as a function of its internuclear distance in the field of a hypothetical tetrahedral water molecule, where the effect of the other hydrogen is neglected, plus the constant energy of two electrons in a tetrahedral hybrid of the other similar hypothetical water

\footnotetext{
${ }^{13}$ S. W. Peterson and H. A. Levy, Acta Cryst. 10, 70 (1957).
} 
TABIE II. SCF-MO of the two lowest MO for some values of $d$ and $D . \phi_{i}=c_{i 1} h_{1}+c_{i 2} h_{2}+c_{i 3} h$.

$q_{1}, q_{2}$, and $q$ are the charges in Atoms $\mathrm{O}_{1}, \mathrm{O}_{2}$, and $\mathrm{H}$ calculated for $d=1.8103$ a.u. by the method of Chirgwin and Coulson.

\begin{tabular}{|c|c|c|c|c|c|c|c|c|c|c|}
\hline$D$ (a.u.) & $d$ (a.u.) & $c_{11}$ & $c_{12}$ & $c_{13}$ & $c_{21}$ & $c_{22}$ & $c_{23}$ & $q_{1}$ & $q_{2}$ & $q$ \\
\hline 4.7 & $\left\{\begin{array}{l}1.8103 \\
2.3500 \\
2.8897\end{array}\right.$ & $\begin{array}{l}0.461 \\
0.177 \\
0.706\end{array}$ & $\begin{array}{l}0.344 \\
0.678 \\
0.043\end{array}$ & $\begin{array}{l}0.531 \\
0.437 \\
0.409\end{array}$ & $\begin{array}{r}-0.457 \\
-0.705 \\
0.502\end{array}$ & $\begin{array}{r}0.897 \\
0.625 \\
-0.811\end{array}$ & $\begin{array}{l}-0.122 \\
-0.344 \\
-0.393\end{array}$ & 1.16 & 1.87 & 0.97 \\
\hline 5.2154 & $\left\{\begin{array}{l}1.8103 \\
2.6077 \\
3.4051\end{array}\right.$ & $\begin{array}{l}0.542 \\
0.173 \\
0.722\end{array}$ & $\begin{array}{l}0.204 \\
0.731 \\
0.023\end{array}$ & $\begin{array}{l}0.540 \\
0.412 \\
0.395\end{array}$ & $\begin{array}{r}0.302 \\
-0.704 \\
0.441\end{array}$ & $\begin{array}{r}-0.957 \\
0.581 \\
-0.849\end{array}$ & $\begin{array}{r}0.054 \\
-0.382 \\
-0.401\end{array}$ & 1.11 & 1.93 & 0.96 \\
\hline 5.7 & $\left\{\begin{array}{l}1.8103 \\
2.8500 \\
3.8897\end{array}\right.$ & $\begin{array}{l}0.572 \\
0.166 \\
0.728\end{array}$ & $\begin{array}{l}0.119 \\
0.778 \\
0.013\end{array}$ & $\begin{array}{l}0.542 \\
0.387 \\
0.389\end{array}$ & $\begin{array}{r}0.195 \\
-0.707 \\
0.394\end{array}$ & $\begin{array}{r}-0.982 \\
0.538 \\
-0.882\end{array}$ & $\begin{array}{r}0.022 \\
-0.418 \\
-0.392\end{array}$ & 1.08 & 1.97 & 0.95 \\
\hline$\infty$ & $\left\{\begin{array}{l}1.8103 \\
2.6077 \\
3.4051\end{array}\right.$ & $\begin{array}{l}0.588 \\
0.641 \\
0\end{array}$ & $\begin{array}{l}0 \\
0 \\
1\end{array}$ & $\begin{array}{l}0.545 \\
0.575 \\
0\end{array}$ & $\begin{array}{l}0 \\
0 \\
0.692\end{array}$ & $\begin{array}{r}-1 \\
-1 \\
0\end{array}$ & $\begin{array}{l}0 \\
0 \\
0.600\end{array}$ & 1.05 & 2 & 0.95 \\
\hline
\end{tabular}

molecule. The case $d=\infty$ then corresponds to the dissociation into ions, one being $\mathrm{H}^{+}$and the other could be considered approximately as $\mathrm{HO}^{-}$. The energy of the process may therefore be assimilated to the protonic affinity of the $\mathrm{HO}^{-}$ion. Rosenfeld ${ }^{14}$ obtains a value of $25.6 \mathrm{eV}$ from a SCF calculation without the assumption of tetrahedral hybrids, which compares well with ours $(31.6 \mathrm{eV})$. The experimental value is $16.6 \pm 0.5 \mathrm{eV}$. Configuration interaction with another closed-shell configuration allows for dissociation into the neutral fragments, $\mathrm{H}$ and $\mathrm{HO}$, and the energy of the process can be considered approximately as the energy of dissociation of a $\mathrm{H}-\mathrm{O}$ bond, giving $13.3 \mathrm{eV}$. The experimental value of Herzberg ${ }^{15}$ for the dissociation of the $\mathrm{H}-\mathrm{O}$ radical is $4.35 \mathrm{eV}$. As the oxygen atom in our calculation remains after dissociation in a tetrahedral configuration, we must subtract about $5 \mathrm{eV}$ for the promotion to the corresponding valence state. We are, therefore, left with a value of about $8 \mathrm{eV}$, still too high.

The energy of the hydrogen bond is obtained as the difference between the energies, $E$, for $D=2.76 \AA$ and $D=\infty$, that is, the difference in energy between two hydrogen-bonded water molecules and two free water molecules. The value obtained is $-0.54 \mathrm{eV}=-12.4$ $\mathrm{kcal} / \mathrm{mole}$, about twice the experimental value obtained from the sublimation energy of ice. If the larger three-center integrals are calculated numerically instead of approximated by Mulliken's procedure, the value reduces to $-8.2 \mathrm{kcal} / \mathrm{mole}$, in better agreement with experiment. The shapes of the curves of Fig. 2 are not altered, only slightly shifted.

In Table II we give the SCF-MO for the two occupied MO for some values of $D$ and $d$. It is seen that the contribution of $h_{2}$ to the lowest MO and of $h_{1}$ and $h$ to the next MO increases with $d$, as expected, thus showing the delocalization of charge (see below for definition of delocalization). The delocalization also increases when $D$ decreases, as expected. The last three columns of Table II give the charges $q_{1}, q_{2}$, and

\footnotetext{
${ }^{14}$ J. L. J. Rosenfeld, J. Chem. Phys, 40, 384 (1964).

${ }^{15} \mathrm{G}$. Herzberg, Spectra of Diatomic Molecules (D. Van Nostrand Company, Inc., New York, 1960).
}

$q$ on Atoms $\mathrm{O}_{1}, \mathrm{O}_{2}$, and $\mathrm{H}$, calculated by the method of Chirgwin and Coulson ${ }^{16}$ and it also shows clearly the effect of the delocalization.

\section{DIFFERENT CONTRIBUTIONS TO THE ENERGY OF THE HYDROGEN BOND}

We define the delocalization energy as the difference between the energy of the hydrogen bond as defined in Sec. 5 and a similar energy obtained with localized orbitals for $D=2.76 \AA$. This localized orbitals are those obtained from the SCF-MO calculation for $D=\infty$. The delocalization energy obtained is $-9.6 \mathrm{kcal} / \mathrm{mole}$ and agrees very well with Tsobomura's ${ }^{3}$ result by the valence bond method.

We define the electrostatic contribution to the energy of the hydrogen bond in a way similar to Ref. 6 as the sum of all the interactions between two water molecules including only the hydrogen involved in the bond, when the charge distributions are replaced by point charges. The molecule centered in $\mathrm{O}_{1}$ (see Fig. 1) is represented by the positive charges +1 in the hydrogen and +6 in the oxygen and three negative point charges. The negative charges are situated in the mean value of the position of the three hybrids not involved in the hydrogen bond, two charges being of -2 and one of -1 according to whether the hybrid corresponds to a lone pair or not. The molecule on center $\mathrm{O}_{2}$ is similarly represented by a positive charge +6 on the oxygen and three negative point charges, one of -2 and two of -1 . The other four electrons (those forming the hydrogen bond) are replaced by two point charges of -2 each, situated in the mean values of the position of the corresponding charge clouds, these being obtained from the SCF-MO calculation for the case $D=\infty$. The value obtained for the electrostatic contribution is $-5.4 \mathrm{kcal} / \mathrm{mole}$.

The Coulombic potential energy of interaction between two hydrogen-bonded water molecules was also computed in a different way. The charge distributions

\footnotetext{
${ }^{16}$ B. H. Chirgwin and C. A. Coulson, Proc. Roy. Soc. (London)
} A201, 196 (1950). 
were not replaced by point charges and the $\mathrm{SCF}-\mathrm{MO}$ for the case $D=\infty$ were used. This calculation is therefore similar to the previous one, but with all the interactions properly calculated. The energy obtained is $-10.6 \mathrm{kcal} /$ mole which shows that one must be careful in considering the effect of the overlap. It increases the repulsion as well as the attraction terms, in such a way that the total effect is attractive.

If one calculates the energy of the hydrogen bond with the same localized orbitals (the SCF-MO for the case $D=\infty$ ) the value obtained is $-2.5 \mathrm{kcal} / \mathrm{mole}$, very different from the previous one of $-10.6 \mathrm{kcal} / \mathrm{mole}$. This energy includes, we believe, all contributions except delocalization. It includes, therefore, the electrostatic contribution, the repulsions and attractions due to overlap, exchange effects, and also some contributions which turn out to be extremely important, namely, the changes in kinetic energy of the electrons and in the intramolecular potential energy, both due, also, to the overlap between the charge clouds of both molecules. For example, the kinetic energy changes from 6.45 a.u. for $D=\infty$ to 6.54 a.u. for $D=2.76 \AA$. Similar changes should occur for the other terms.

These important changes in terms which we believe were not considered up to now show the difficulty of calculating the energy of the hydrogen bond as the sum of four different contributions: electrostatic, delocalization, repulsion, and dispersion. ${ }^{4}$ We consider that only the electrostatic contribution and the delocalization energy are rather well-defined terms, the other two not being simply related to a quantummechanical calculation as the present one.

\section{DIPOLE MOMENT}

Due to hydrogen bonding there is a delocalization of charge which produces a net migration of charge from the $\mathrm{O}_{2}$ region to the $\mathrm{O}_{1}$ region, and a corresponding increase in the dipole moment.

The dipole moment in gaseous water and in ice was calculated by computing the contributions of the two $\mathrm{O}-\mathrm{H}$ bonds, $\mu_{B}$, and of the two lone pairs, $\mu_{L}$, to the total dipole moment of a $\mathrm{H}_{2} \mathrm{O}$ molecule, $\mu$. We have used the SCF-MO for $D=\infty$ and $D=2.76 \AA$ for free water and ice, respectively, and the corresponding
TABLE III. Dipole moments in debyes.

\begin{tabular}{lrr}
\hline \hline & Free water & \multicolumn{1}{c}{ Ice } \\
\hline$\mu$ & 1.68 & 2.40 \\
$\mu_{B}$ & -1.54 & -3.25 \\
$\mu_{L}$ & 3.22 & 5.65 \\
\hline \hline
\end{tabular}

dipole moments so obtained are 1.68 and $2.40 \mathrm{D}$, respectively. This last value agrees extremely well with recent results of Eisenberg ${ }^{17}$ of $2.40-2.87 \mathrm{D}$, and also fairly well with that of Onsager and Dupuis ${ }^{18}(3 \mathrm{D})$, and confirms the idea that the dipole moment in ice is definitely about 1.5 times that of gaseous water.

It should be noted (see Table III) that due to our use of tetrahedral hybrids directed along tetrahedral directions the dipole moment $\mu_{L}$ is very high, and to compensate this value, $\mu_{B}$ results of opposite sign, in contradiction with the results obtained for the free water molecule by Burnelle and Coulson ${ }^{19}$ from the wavefunctions of Ellison and Shull. ${ }^{5}$ Comparison of Burnelle and Coulson's results with ours also indicate that although the total dipole moment of water is not very sensitive to changes in the geometry and in the wavefunctions, each of its contributions, $\mu_{L}$ and $\mu_{B}$, vary appreciably with them.

When hydrogen bonding is produced, the delocalization of charge is such that both $\mu_{L}$ and $\mu_{B}$ increase, the former one increasing much more, thus producing a net increase in dipole moment $\mu$.

\section{ACKNOWLEDGMENTS}

We are very grateful to the Laboratory of Molecular Structure and Spectra of the University of Chicago for providing all exchange and hybrid integrals and to the Instituto de Cálculo de la Universidad de Buenos Aires for generous allowance of machine time.

We thank Dr. Alicia Batana for reading the manuscript.

${ }^{17}$ D. S. Eisenberg, Progress Report, Session 1962-1963, Mathematical Institute, University of Oxford, p. 69.

${ }^{18}$ L. Onsager and M. Dupuis, Rend. Soc. Stal. Fis. Corso X, p. $294(1960)$.

${ }^{19}$ L. Burnelle and C. A. Coulson, Trans. Faraday Soc. 53, 403 (1957). 